\title{
Simulation and the experimental test of radial-deformation of spindle-tool-holder connection system at high-speed
}

\author{
Lan $\mathrm{JIN}^{1, \mathrm{a}}$, Li Zhao ${ }^{2, \mathrm{~b}}$, Liming XIE ${ }^{1, \mathrm{c}}$, Zhiyuan $\mathrm{RUI}^{1, \mathrm{~d}}$ and Dongxing $\mathrm{SHI}^{3, \mathrm{e}}$ \\ ${ }^{1}$ Key Laboratory of Digital Manufacturing Technology and Application, Ministry of Education, \\ Lanzhou University of Technology, Lanzhou, China \\ ${ }^{2}$ School of Mechanical \& Electronical Engineering, Lanzhou University of Technology, Lanzhou, \\ China \\ ${ }^{3}$ Wuhan Marine Machinery Plant Co,.Ltd, Wuhan, China \\ alan-jane@126.com, bzhaoli.zh@qq.com, ${ }^{\mathrm{c} x i e l m 810 @ s i n a . c o m, ~}{ }^{\mathrm{d}}$ zhiy_rui@163.com, \\ eshi409878118@163.com
}

\begin{abstract}
Keyword: High-speed spindle, Radial-deformation measurement, 7:24 Tapered spindle-tool-holder, Dynamic real-time experimental method.

Abstract. A dynamic real-time method is proposed in this paper for measuring the spindle-end radial-deformation under spindle speed of 10000 15000 r/min. The system consists of three non-contact eddy current sensors and a laser deformation sensor used to measure the radial-deformation of the rotating simulating tool, and the experimental data was analyzed with the data analysis subsystem, computer and LMS Test. Lab. Experimental studies show that this system could be used to measure the spindle radial-deformation. It demonstrates that the simulated results is consistency with the experiment results. And it is also verified the feasibility of the 7:24 tapered spindle-tool-holder used for the high-speed machining tool under speed of $15000 \mathrm{r} / \mathrm{min}$.
\end{abstract}

\section{Introduction}

It is evident that today's machine tools are facing demands for increased power and speed, as shown by Ashok and Samuel [1]. The rapid development of the modern manufacture in producing high-speed motorized spindle could be observed from the area of machine tools application [2]. The working performance of machine tool is conditioned by the performance of certain vital assemblies. Particularly predominant are the spindle units, as the parameter units of machine tools, since they determine the accuracy and productively [3]. It is not only influences the machining precision of machine tools but also geometrical shape and surface roughness of work-piece [4]. Spindle-end radial-deformation is a variation of the actual axis of the spindle relative to ideal axis in the measurement plane within the specified. During high-speed machining, the radial expansion of spindle-tool-holder connection system under the centrifugal force could present. The expansion of spindle-end is always greater than that of the tool-holder, which causes clearance between connection surfaces of spindle-tool-holder. The existence of clearance at the connection not only decreased the contact stress of spindle-tool-holder connection, but caused an offset of tool-holder contrary to spindle, resulting in tool-holder bending under radial cutting forces, and seriously affecting the machining accuracy and surface roughness. Thus, the identification of the spindle-end radial-deformation under the high-speed has become very important.

In an earlier work, $\mathrm{Cao}$ and $\mathrm{Li}$ et al [5] considered the centrifugal expansion of rotating components, a dynamic model for the high-speed spindle bearing was given with experimental verification. In order to take the rotation accuracy of the spindle as a important point for achieving the desired form accuracy and surface roughness of machined component [6]. After this, many machine tool testing methods were adopted in many international standards such as American National Standards Institute (ANSI) and ISO, and ANSI especially drew up the standard for the test of the spindle [7]. Especially, radical deformation of the spindle is recognized as a significant source of error that affects the accuracy of the machine tools [8]. With the development of spindle researches, the thermal and mechanical behaviors of high-speed motorized spindles have become very difficult to 
predict for spindle designer and users [9]. Udup and Bisu [10] evaluated the temperature distribution and its effects on the axial and spindle radial-deformation, and designed a simulation procedure to simulate two numerical models to research this issue.

More important is to measure the deformation of the high-speed spindle system under working conditions. Many different methods and model of spindle deformation measurements have been provided [11-13]. As the rotation speed of a machining center spindle is in the order of $15000 \mathrm{r} / \mathrm{min}$, high-frequency response sensing methods are required for spindle error measurement [14]. Laser-based measurement techniques could meet the needs of the measurements and have already been used [15,16], but it requires accurate calibration as the output characteristics of the laser beam is nonlinear with respect to the deformation of spindle due to the reflectivity of the target surface installed in the spindle [1]. Eddy current sensor perfectly meets the high-speed and accuracy requirements of spindle radial-deformation measurement. In most instances, the spindle radial-deformation measurement is affected by inherent error sources such as sensor offset, thermal drift of spindle, centering error, and form error of the target surface installed in the spindle [17]. Various processing and measuring methods were suggested by the researchers to eliminate the unwanted contributions in spindle error data. However, it is difficult to implement these methods in a machining center due to space limitations and high-speed conditions.

In this paper, a dynamic real-time experiment for radial-deformation of spindle-tool-holder measurement is proposed and the corresponding experimental results are reported. After analyzing of experimental results and ANSYS simulation results, the feasibility and accuracy of the test method is further demonstrated the analysis of 7:24 tapered spindle-tool-holder connection, and this paper also suggests two methods for improving 7:24 tapered spindle-tool-holder connection performance.

\section{FEA Analysis of the spindle-tool-holder connection}

\section{Forces Analysis of Spindle-tool-holder Connection}

The centrifugal force of the tool system increases gradually as the speed increases, when the speed reaches over $8000 \mathrm{r} / \mathrm{min}$, the centrifugal force will become the main load on the tool system [18]. The spindle and tool-holder are only affected by the radial centrifugal force under constant angular velocity. This is regarded as a static problem and its shear stress component is zero [19]. The tangential rigid deformation component caused by the rotation of the spindle and tool-holder could be ignored, and only relative deformation part is considered in the analysis, thus the single-valued condition of deformation component could be ensured. Therefore, the radial centrifugal force regarded as the radial force act on the spindle and tool-holder, the problem could be solved as an axisymmetric plane stress problem.

For the material of spindle is very similar with tool-holder's, it is assumed that the materials of spindle and tool-holder are identically elastic-plastic behavior in this paper. Fig.1(a) is BT50 spindle-tool-holder connection simplified structural illustration without considering some details such as the keyway, and Fig.1(b) is the cross section shape of spindle-tool-holder connection.
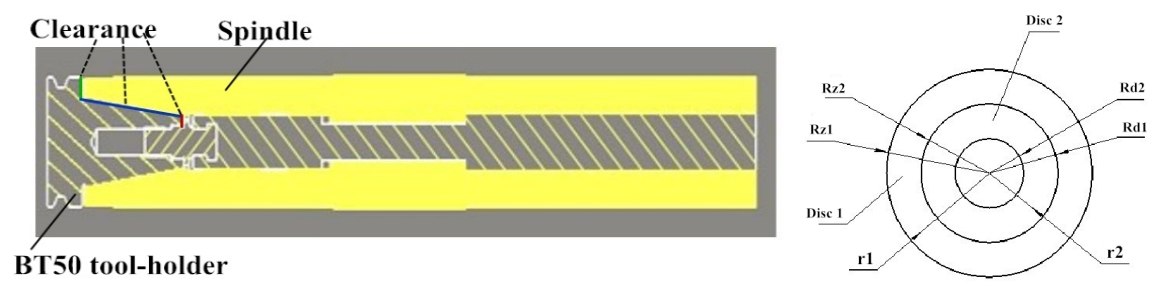

Fig.1. 7:24 tapered spindle-tool-holder connection and cross section shape

The equilibrium equation of rotating simulating tool, which is considered as the tool mounted on the spindle, could be expressed by the following Eq. 1 [20]. 


$$
\frac{d \sigma_{r}}{d r}+\frac{\sigma_{\theta}}{r}+\rho \omega^{2} r=0
$$

Where $\sigma_{\mathrm{r}}$ is the radial stress, $\sigma_{\theta}$ is the tangential stress, $\rho$ is the material density, $\omega$ is the angular speed around the central axis.

The elastic constitutive equation is performed from Eq. 2.

$$
\left.\begin{array}{l}
\varepsilon_{\theta}=\frac{1}{E}\left(\sigma_{\theta}-v \sigma_{\mathrm{r}}\right) \\
\varepsilon_{\mathrm{r}}=\frac{1}{\mathrm{E}}\left(\sigma_{\mathrm{r}}-v \sigma_{\theta}\right)
\end{array}\right\}
$$

Where $v$ is the Poisson ratio, $E$ is modulus of elasticity. And the geometric equation could be expressed by Eq. 3 .

$$
\varepsilon_{\mathrm{r}}=\frac{\mathrm{du}}{\mathrm{dr}}, \varepsilon_{\theta}=\frac{\mathrm{u}}{\mathrm{r}}
$$

Therefore, the stress component can be obtained based on the above formulas as following.

$$
\left.\begin{array}{c}
\sigma_{r}=C_{1}+\frac{C_{2}}{r^{2}}-\frac{3+v}{8} \rho \omega^{2} r^{2} \\
\sigma_{\theta}=C_{1}-\frac{C_{2}}{r^{2}}-\frac{1+v}{8} \rho \omega^{2} r^{2}
\end{array}\right\}
$$

The radial-deformation of the rotating simulating tool, which is deduced from the elastic constitutive equation and the geometric equation, are given by Eq. 5 .

$$
u(r)=r \varepsilon_{\theta}=\frac{r}{E}\left[\sigma_{\theta}-v \sigma r\right]
$$

Based on the aforementioned, the deformation amount of simulating tool at any radius could be calculated as Eq. 6.

$$
u(r)=\frac{r}{E}\left[(1-v) C_{1}-\frac{C_{2}}{r^{2}}(1+v)-\frac{1+3 v}{8} \rho \omega^{2} r^{2}+\frac{(3+v)}{8} v \rho \omega^{2} r^{2}\right]
$$

Where $C_{1}$ and $C_{2}$ is two constants.

The clearance between the spindle and tool-holder is zero in working condition. Regardless of the shrink range that is caused by cone connection fit, the boundary conditions are Eq. 7 and 8.

$$
\begin{aligned}
& \left.\sigma_{r}\right|_{r=R_{\mathrm{Z1}}}=0,\left.\sigma_{r}\right|_{r=R_{\mathrm{Z} 2}}=0 \\
& \left.\sigma_{r}\right|_{r=R_{d 1}}=0,\left.\sigma_{r}\right|_{r=R_{d 2}}=0
\end{aligned}
$$

Where Eq. 7 is the values for the stress carried by spindle's inside radius and it's outside radius, and Eq. 8 is the values for the stress carried by tool-holder's inside radius and it's outside radius.

The boundary conditions are substituted into Eq. 4 and Eq. 6 to calculate the radial-deformation components. When the spindle and tool-holder rotate around its central axis by isometric speed $\omega$, under the action of the radial centrifugal force $I_{r}=\rho \omega^{2} r$, the radial-deformation components of the spindle-tool-holder connection interface, $u_{t}(a)$ and $u_{s}(a)$, could be expressed as Eq.9 and 10.

$$
u_{t}(a)=\frac{\left(3-2 v_{t}\right)\left(1+v_{t}\right)}{8 \mathrm{E}_{t}\left(1-v_{t}\right)} \rho_{t} \omega^{2} a\left[\left(1-2 v_{t}\right) a^{2}+\frac{2 v_{t}-1}{3-2 v_{t}} a^{2}\right]
$$




$$
u_{s}(a)=\frac{\left(3-2 v_{s}\right)\left(1+v_{s}\right)}{8 E_{s}\left(1-v_{s}\right)} \rho_{s} \omega^{2} a\left[b^{2}+\left(1-2 v_{s}\right)\left(a^{2}+b^{2}\right)+\frac{2 v_{s}-1}{3-2 v_{s}} a^{2}\right]
$$

Where $u_{t}$ and $u_{s}$ are the spindle radial-deformations and tool-holder radial-deformations, $a$ is the spindle-tool-holder connection radius for any sections ( is the size between tool-holder external diameter and spindle internal diameter), $b$ is the spindle external diameter, $v_{t}$ and $v_{s}$ are the spindle radius and tool-holder radius, $E_{t}$ and $E_{s}$ are the spindle elastic modulus and tool-holder elastic modulus, $\omega$ is angular velocity $\left(\omega=2 \pi n / 60, n\right.$ is rotation rate), $v_{t}$ and $v_{s}$ are the spindle Poisson ratio and tool-holder Poisson ratio, $\rho_{t}$ and $\rho_{s}$ are the spindle density and tool-holder density.

The spindle and tool-holder are made of steel material, their elastic modulus, Poisson ratio and density are almost equal. Thus the radial gap between the spindle and tool-holder is Eq.11.

$$
\delta=2\left(u_{s}-u_{t}\right)=\frac{(3-2 v)(1+v)}{2 E} \rho \omega^{2} a b^{2}>0
$$

For the spindle-tool-holder connection system, the spindle radius $b$ and tool-holder radius a are the constants. If the material of the spindle and tool-holder is selected, the elastic modulus, Poisson ratio and density could have been fixed. In the machining process, the radial-deformation of the spindle-tool-holder connection and the clearance between them is only related to the rotation rate, and the clearance between the spindle and tool-holder increases with the spindle rotation rate.

As shown in Fig.1, the radial clearance would change with the connection radius if the angular velocity could be constant, and it makes the connection clearance of the big-end bigger than that of the small-end. Therefore, in the clearance calculation, the connection clearance of the small-end must meet the permission scope as long as the connection clearance of the big-end is within the allowable range for using it.

\section{FEA Model of the Spindle-tool-holder}

Under high rotation speed, the spindle and tool-holder experience the stresses caused by interference fit and the centrifugal stress [21]. The spindle-tool-holder connection belongs to the complex contact problem of nonlinear boundary condition. The interface presents complicated contact state and stress state. The finite element method and ANSYS analysis software are used in this paper to establish flexible-flexible contact analysis. The contact boundary conditions could be determined through analysing nodes on the target surface as well as the relationship between degrees of freedom of the contact surface and the consistency of deformation. Then baised on the variational principle of the boundary deformation coordination we start to simulate the complex contact surface problems and dynamic contact problems.

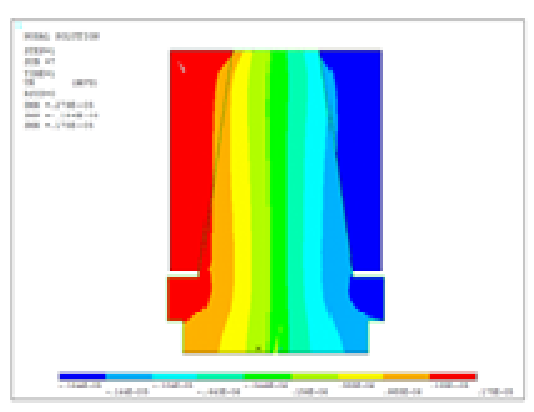

(a)

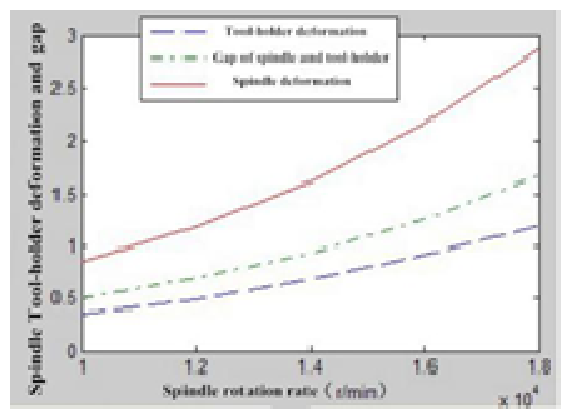

(b)

Fig.2. The spindle-tool-holder radial-deformation at $\mathrm{N}=10000 \mathrm{r} / \mathrm{min}-18000 \mathrm{r} / \mathrm{min}$, and interface gap

Fig.2 (a) shows the radial-deformation of the spindle-tool-holder connection at the rotating speed of $15000 \mathrm{r} / \mathrm{min}$ and the drawbar force of $15000 \mathrm{~N}$. In Fig.2 (b)The spindle-tool-holder connection of the radial-deformation diagram at the maximum diameter is obtained through ANSYS analysis for the spindle speed of 10000-18000 r/min.

It can be seen from Fig.2 (a) that the maximum radial deformation of the spindle taper hole at 
maximum radial is $1.86 \mu \mathrm{m}$, and the maximum radial deformation of the tool-holder is $1.44 \mu \mathrm{m}$ at maximum radial. So the gap between spindle and tool-holder is $0.42 \mu \mathrm{m}$. it also can be seen from Fig.2 (b) that the gap increases with spindle speed nonlinearly and the maximum gap at spindle speed 18000 $r / m i n$ is about $1.65 \mu \mathrm{m}$. The simulated results shows that 7:24 taper holder at pull force $\mathrm{N}=15 \sim 18 \mathrm{kN}$ could be used in the high speed spindle machining tool at $15000 \mathrm{r} / \mathrm{min}$ and even more.

\section{Measurement principle}

The radial-deformation of the spindle-tool-holder is needed to be measured to verify the simulation results. However, the tool-holder is assembled in taper hole of spindle, so the deformation of the tool-holder and the small-end of spindle is impossible to be directly measured, the only way to verify the simulation results is to measure the radial-deformation of spindle at the big-end and then to evaluate the gap at the small end according to the model. Furthermore, the speed range suitable to high-speed machining of spindle-tool-holder system could be obtained by testing the spindle radial-deformation.

A dynamic real-time experiment for spindle radial-deformation measurement at the speed range of $10000 \sim 15000 \mathrm{r} / \mathrm{min}$ is proposed in this section to verify the simulation results of spindle-tool-holder system and furthermore to confirm what is the suitable high-speed range for the spindle-tool-holder system to be at a good working condition.

\section{Sensors distribution}

A dynamic real-time experiment for spindle radial-deformation measurement is described with Four-point method. In present work, simulating tool was used to reflect the performance of spindle-tool-holder connection. The experiment system for the radial-deformation measurement of the spindle-tool system is shown in Fig.3.

In one measuring route, the installation of the sensor 1 and 2 is shown in Fig.3 (b). For measuring rotation accuracy of spindle, three MICRO-EPSILON eddy NCDT 3010sensors, which is a non-contacting displacement measuring system operating on the eddy current principle, with $0.1 \mu \mathrm{m}$ of static repeatability, made in Germany and used for measuring targets made of electrically conductive materials that may be either ferromagnetic or non-ferromagnetic, are mounted in orthogonal directions on a mounting bracket which is installed on the spindle housing. The sensors 1 and 2 are maintained at the same distance of their surface to the simulating tool [4], and the sensor 3 measures axial center deformation of simulating tool to be installed on the axial spindle center. Fig. 4 is the test bench for spindle radial deformation. Under no-load running state, the spindle axial deformation could be measured through deformation change between the eddy current sensor and the simulating tool. The change of distance between the probe coil and the simulating tool is transformed by eddy current sensor into the change of three parameters which are equivalent inductance of the coil, equivalent impedance and quality factors. These three parameters could be converted into voltage signal by adding corresponding preamplifier, and then transferred to the computer through $A / D$ converter to be processed through LMS.Test.lab to get the deformation digital signal of spindle-tool system.

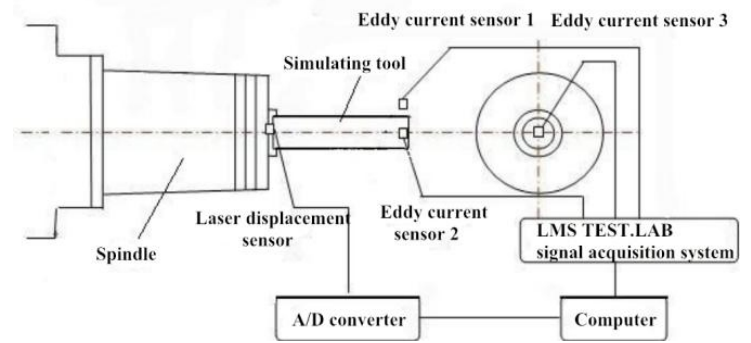

(a)

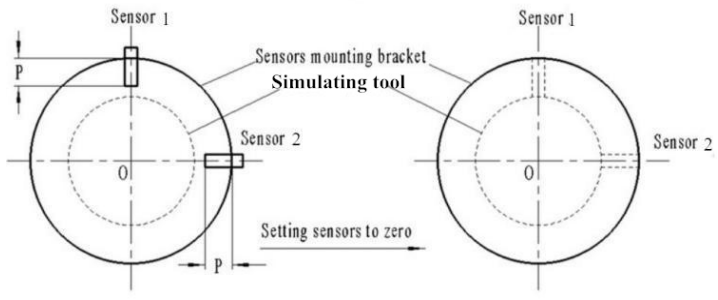

(b)

Fig.3. Spindle radial deformation measurement system

In another measuring route, the installation of laser deformation sensor is shown in Fig.4. One KEYENCE LK-G80 laser sensor with $0.1 \mu \mathrm{m}$ of repeatability accuracy and $\pm 0.25 \%$ F.S of linearity, made in Japan, is mounted on the spindle-end for measuring radial-deformation of spindle-tool-holder. 
The measured deformation data is processed by the LK-Navigator software, and the measurement of deformation of the simulating tool could be calculated into the deformation of tool-holder's.

Finally, the computer synthesizes the two sets of dates that are collected from two measuring routes in the same experimental step.
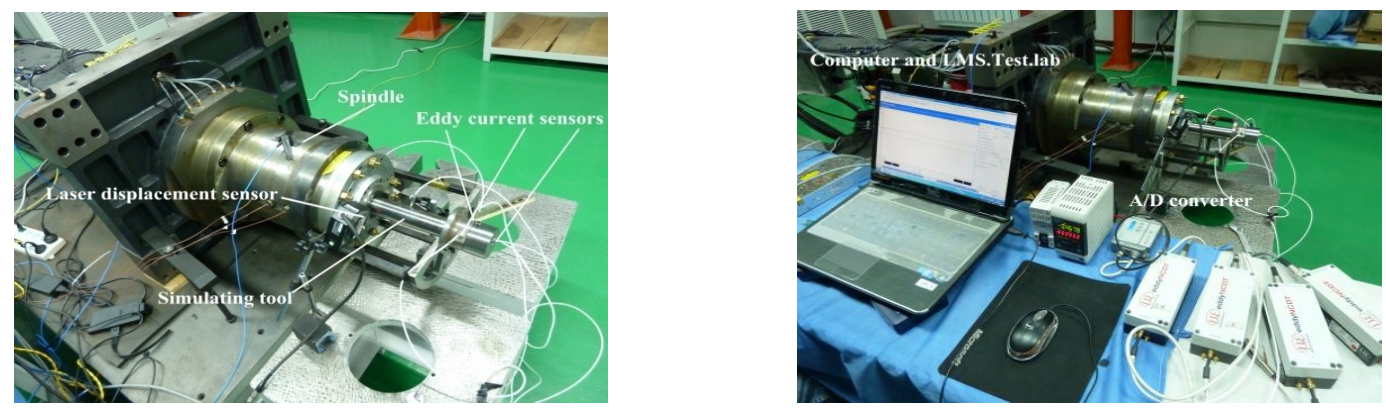

Fig.4. the installation of laser deformation sensors

\section{Process and Analysis of Spindle Radial-deformation}

The experimental results of spindle radial-deformation are shown in Tab.1. It shows that the radial vibration of the spindle is smaller than $3 \mu \mathrm{m}$ which is allowed by the precision machine tools. Therefore, within the speed range of 10000 15000 r/min it also shows that 7:24 spindle-tool-holder connection can be used to high-speed machining of $15000 \mathrm{r} / \mathrm{min}$.

Tab.1 The experimental radial-deformation of the spindle (unit: $\mu \mathrm{m}$ )

\begin{tabular}{cccccc}
\hline $\begin{array}{c}\text { Spindle speed } \\
(\mathrm{r} / \mathrm{min})\end{array}$ & 10000 & 11000 & 12000 & 14000 & 15000 \\
\hline $100 \mathrm{~s}$ & 1.502 & 0.767 & 0.979 & 0.832 & 2.232 \\
\hline $200 \mathrm{~s}$ & 1.888 & 0.997 & 1.41 & 1.59 & 2.216 \\
\hline $300 \mathrm{~s}$ & 2.368 & 1.073 & 1.79 & 1.24 & 2.197 \\
\hline $400 \mathrm{~s}$ & 0.908 & 1.399 & 1.09 & 1.26 & 2.157 \\
\hline $500 \mathrm{~s}$ & 1.820 & 1.37 & 1.42 & 1.58 & 2.113 \\
\hline
\end{tabular}

Due to there probably exist the interference factors, including environment, working conditions and other man-made reasons in the measuring process, and it could not be ignored. And the spindle radial-deformation results include the thermal deformation and self-excited vibration deformation, the former is caused by spindle temperature rise which is generated through inner motor and bearings; the latter is caused by high-speed. For these reasons and based on the experimental results, although the testing results are larger than the spindle radial-deformation simulation results caused by spindle radial centrifugal force, the experimental result could visually reflects the characteristics of the spindle radial-deformation, and the changing trend of the experiment and simulation is accordant. So, the spindle-tool-holder connection radial-deformation simulation result is correct, and practicability of the experimental method in measuring spindle radial-deformation is further explained.

\section{Conclusion}

This study has used finite element method to analyze the spindle-tool-holder connection characteristics varied with rotation speed, as well as the deformation rule of spindle-tool-holder connection. 
The study shows that the axial tension should be increased appropriately to enhance the magnitude of interference and coupling stress between contact surface, therefore the reliability and processing quality of 7:24 tapered spindle-tool-holder could be improved. ANSYS results of the gap between spindle-tool-holder connection interfaces verify that 7:24 taper tool-holder could be used for high-speed machining center spindle-tool connection with the spindle-speed of $15000 \mathrm{r} / \mathrm{min}$, and whirling speed of 7:24 taper tool-holder is $18000 \mathrm{r} / \mathrm{min}$ at the the drawbar $\mathrm{F}=15 \sim 18 \mathrm{KN}$. With increasing spindle rotation speed, the processing value of big-end of spindle taper is $0.5 \mu \mathrm{m}$ lower than standard 7:24 spindle taper big-end, thus could make up for the spindle orifice expansion caused by high-speed centrifugal force, then reduce the clearance between the spindle and tool-holder, and improve spindle-tool-holder coupling performance.

According to the proposed dynamic real-time experimental method for spindle radial-deformation measurement, the spindle radial-deformation for spindle-tool system is tested at 10000 15000 $/ \mathrm{min}$. The test results compared with ANSYS simulation result demonstrate the feasibility and accuracy of the test method.

\section{Acknowledgement}

This reserch was financially supported by the the National Science and Technology Major Project (2010ZX04001-032).

\section{References}

[1] S. D. Ashok, G. L.Samuel, Modeling, measurement, and evaluation of spindle radial errors in a miniaturized machine tool. Int J Adv Manuf Technol, vol. 59, Mar (2012) 445-461.

[2] Abele E, Altintas Y, Brecher C. Machine tool spindle units, CIRP Ann Manuf Technol, vol. 59, Mar (2010) 781-802.

[3] Zverev I. A, Eun I-U, Chung W. J, Lee C M. Simulation of spindle units running on rolling bearings. Int J Adv Manuf Technol, vol. 21 (2003) 889-895.

[4] Lan Jin, Zhaoyang Yan, Liming Xie et al. An experimental investigation of spindle rotary error on high-speed machining center. Int J Adv Manuf Technol, vol. 70 (2012) 327-334.

[5] Cao Hong rui, Li Bing, Chen Xuefeng, et al. Centrifugal expansion of high-speed spindle and its influences on bearing dynamic characteristics. Journal of Mechanical Engineering, vol. 48, Oct (2012) 59-64.

[6] Bryan J, Clouser R, Holland E. Spindle accuracy. American Machinist/Metalworking Manufacturing, v 111, n 25, p 149-164, Dec 4, 1967.

[7] Jyweb W-Y, Chen C-J. The development of a high-speed spindle measurement system using a laser diode and a quadrants sensor. Int Mach Tool, vol. 45, Aug (2005) 1162-1170.

[8] Uriarte L, Herrero A, Zatarain M, Santiso G. Error budget and stiffness chain assessment in micro milling machine equipped with tools less than $0.3 \mathrm{~mm}$ in diameter. Precis Eng, vol. 31, Jan (2005)1-12.

[9] Lin C-W, Tu JF, Kamman J. An integrated thermo-mechanical-dynamic model to characterize motorized machine tool spindles during very high-speed rotation. Int Mach Tool, vol. 43, Aug (2003) 1035-1050.

[10] Udup Emil, Bisu Claudiu Florinel, Zapciu, Miron. Evaluation and improvement of the spindle thermal transfer, Applied Mechanics and Materials, vol. 436, Oct (2013)225-232.

[11]Fujimaki K, Sase H, Mitsui K. Effects of sensor noise in digital signal processing of the three-points method. Measurement Science and Technology, vol. 19, Jan (2008) 262-266. 
[12] Shinno H, Mitsui K, Tatsue Y, Tanaka N, Omino T, Tabata T. A new method for evaluating error motion of ultra-precision spindle. CIRP Annals 1987: Manufacturing Technology, Technische Rundschau, Dec. (1987 ) 381-384.

[13]Lee E S,Wi H G. Comprehensive technique for measuring the three-dimensional positioning accuracy of a rotating object. Int J Adv Manuf Technol, vol. 14, July (1998) 330-335.

[14]Liu C H, Yywe W Y, Lee H W. Development of a simple test device for spindle error measurement using position sensitive detector, Meas Sci Technol, vol. 15, Sept(2004) $1733-1741$.

[15] Castro H. F. F, A method for evaluating spindle rotation errors of a machine tool using a laser interferometer, Measurement, vol. 41, June (2008) 526-537.

[16] Min B K, O’Neal G, Koren Y, Pasek Z. A smart boring tool for process control. Mechatronics, vol. 12, Nov (2001) 1097-1114.

[17] Marsh ER. Precision spindle metrology. DE Stech Publications Inc, Lancaster, PA 17602, U.S.A, (2008).

[18]López de Lacalle LN, Lamikiz A. Machine tools for high performance machining, Springer 228, vol.228 (2009).

[19]Zhang Song, AI Xing, Liu Zhanqiang, Tang Weixiao. Finite element analysis of non uniform stress field of 7:24 spindle/toolholder interface. Tool Engineering, vol. 7, June (2003) 25-27.

[20] Wang Jian. Design of high-speed motorized spindle and the analysis of joining performances for tool-holder/spindle interfaces. Dissertation for the Master Degree, Harbin Institute of Technology, (2009) 20-22.

[21]Xie Liming, Yan Zhaoyang, Jin Lan et al. Dynamic test and analysis of high-speed spindle rotation error. Manufacturing technology and machine tools, vol. 4, Apr. (2012) 93-95. 